\title{
Strain measurements in industrial applications: A case study of solder bumps in semiconductor devices
}

\author{
Pawel Nowakowski, Mary Ray and Paul Fischione
}

\section{E.A. Fischione Instruments, Inc., United States}

Understanding strain and strain distribution in industrial materials is critical when studying phenomena such as stress fatigue of welds or solder junctions. The microelectronics fields, in particular, struggle with fatigue problems that can accrue under cyclic thermo-mechanical loading. The presence of elastic and plastic strain can lead to the development of a variety of defects, such as fracture and delamination. Ultimately, these defects can lead to microelectronic device failures.

Many techniques have been developed for plastic and elastic strain study [1-3]. In microscopy and microanalyses, one of the techniques used for deformation structure characterization is electron backscatter diffraction (EBSD).

EBSD plastic strain measurement techniques rely on recording changes in EBSD pattern quality [4, 5] or local changes of crystal orientation within a grain $[6,7]$. The EBSD technique has recently been applied to elastic strain measurements. This approach is based on cross-correlation analysis of high-resolution EBSD (HR-EBSD) patterns. The technique measures lattice distortion-related differences between a reference EBSD pattern and a pattern of interest, both of which are obtained from the same grain [8-10]. Strain studies by EBSD and HR-EBSD are sensitive to diffraction pattern quality [11]. Aside from intrinsic instrumentation limitations, sample preparation factors prominently into the accuracy and precision attained in strain analyses [12-15]. Surface contamination, oxidation, crystal lattice damage, and plastic deformation must be avoided during sample preparation so that accurate and precise measurements can be obtained. A sample preparation technique that preserves the native state of the material microstructure, without introducing artifacts, is necessary to fully understand the failure mechanism. It was reported that the average elastic strain measured by HR-EBSD can be up to 2 orders of magnitude lower for a sample prepared by broad ion beam (BIB) milling when compared to mechanical polishing preparation (MP) [16].

In this presentation, we focus on solder bump cross-section sample preparation and strain measurement. Two different sample techniques are described and compared: conventional MP using colloidal silica suspension and BIB milling. Sample preparation is then confirmed by advanced structural characterizations and strain measurements, which are also explained in detail.

Figure 1 shows strain fields obtained from HR-EBSD measurement of principal strain components $\mathrm{e}_{\mathrm{xx}}$, $e_{y y}$, and $e_{z z}$ in cross-section samples of a nonloaded solder joint (Figure 1a) and a loaded solder joint (Figure 1b). The average strain for a nonloaded device is $10^{-5}$ order of magnitude (Figure 1a), while for a loaded device, the average strain is $10^{-3}$ order of magnitude (Figure 1b). Strain is uniformly distributed around the zero value of the scale in a nonloaded solder bump. Strain is higher - up to 2 orders of magnitude and primarily localized at the solder bump/copper pad interface - in a loaded solder bump. High positive strain presents in a normal $\left(\varepsilon_{\mathrm{zz}}\right)$ direction, a compressed (negative) strain in the horizontal $\left(\varepsilon_{\mathrm{xx}}\right)$ direction, and a mixed strain in the transverse $\left(\varepsilon_{\mathrm{yy}}\right)$ direction. This indicates that during the soldering process, complex loading conditions acted on the solder bump between the package substrate on one side 
and the board on the other side. HR-EBSD measurements confirm the presence of strain, which can lead to cracks or delamination over the course of the device's life.

A structural evolution resulting from cyclic thermo-mechanical loading is shown in Figure 2 (marked by a black rectangle). The $\mathrm{Cu}$ grains in this area have an elongated shape and a preferred $\{110\}$ crystallographic orientation (Figure 2a). This microstructure change is associated with higher crystallographic local misorientation (Figure $2 \mathrm{~b}$ ) caused by elevated plastic strain.

Understanding strain characteristics is the first step toward understanding the failure mechanism and, ultimately, how to prevent these types of failures in the future.

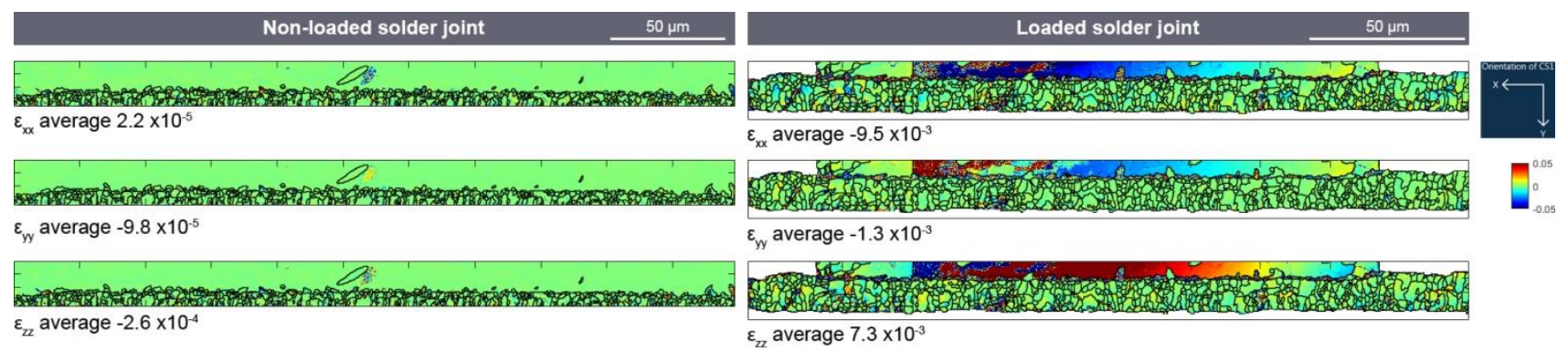

Figure 1. High resolution electron backscatter diffraction strain field obtained for principal strain components exx, eyy, and ezz in a nonloaded solder joint (a) and a loaded solder joint (b) after argon broad ion beam milling. The loaded solder joint shows strain accumulated at the interface of the solder bump and copper pad, as well as at the area between the solder bump void and copper pad.
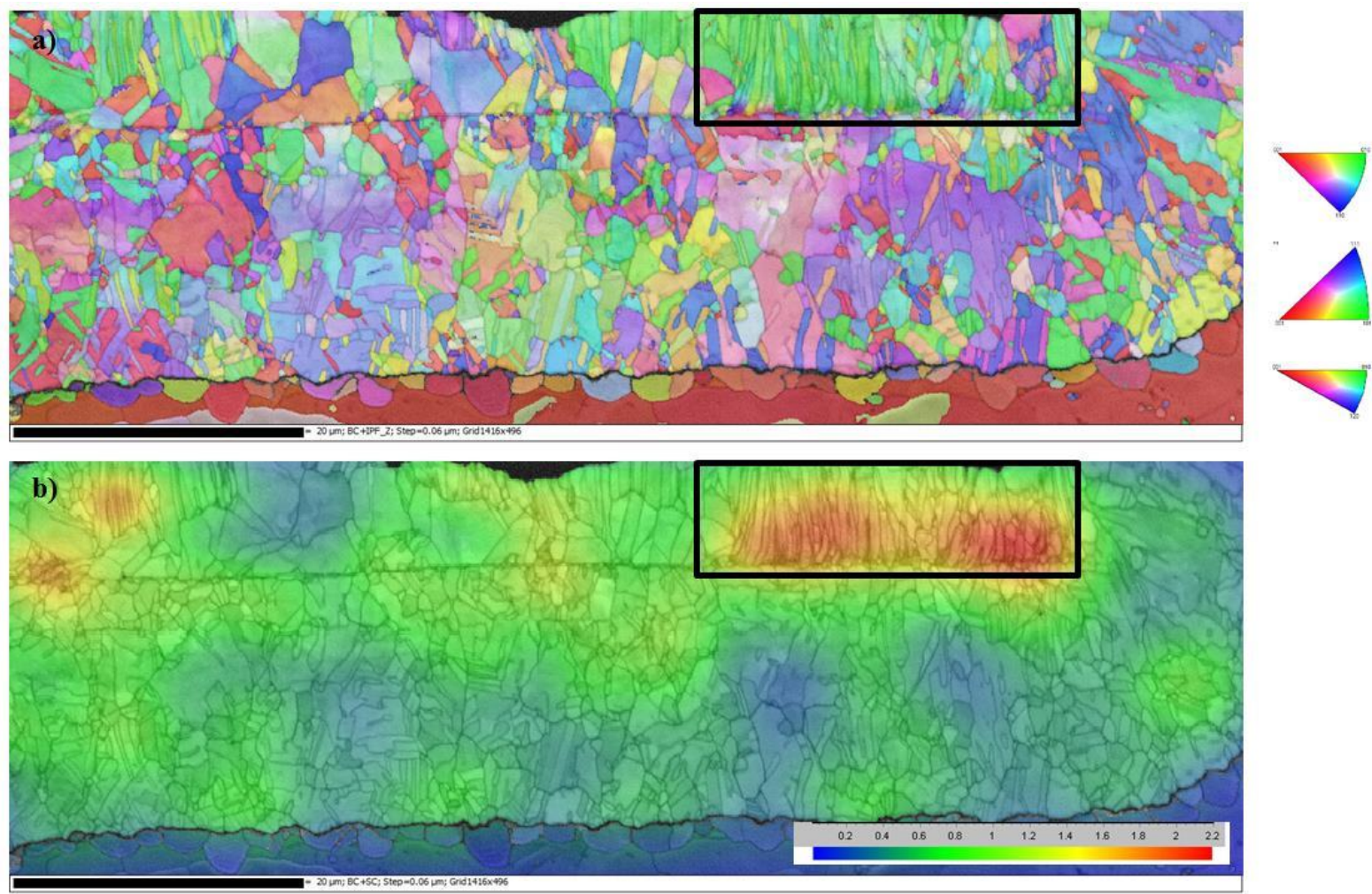
Figure 2. Electron backscatter diffraction maps showing the structural evolution of copper grains (area marked by a black rectangle) due to cyclic thermo-mechanical device loading. Inverse pole figure EBSD color coded map in transversal direction overlaid on band contrast map (a); strain contouring color-coded EBSD map overlaid on band contrast map (b).

References

- Wilkinson, A., \& Dingley, D. (1991). Acta Metallurgica et Materialia, 39(12), 3047-3055.

- Kaboli, S., Demers, H., Brodusch, N., \& Gauvin, R. (2013). Microscopy and Microanalysis, 19(S2), 1756-1757.

- Vaudin, M., Stan, G., Gerbig, Y., \& Cook, R. (2011). Ultramicroscopy, 111(8), 1206-1213.

- Wilkinson, A. J., Gonzalez, G., \& Dingley, D. J. (1993). Journal of Microscopy, 169(2), 255-261.

- Kamaya, M., Wilkinson, A. J., \& Titchmarsh, J. M. (2005). Nuclear Engineering and Design, 235(6), 713-725.

- A. J. Schwartz, M. Kumar, \& B. L. Adams (Eds.), Electron backscatter diffraction in materials science (2nd ed., pp. 247-264). Boston: Springer.

- Ørsund, R., Hjelen, J., \& Nes, E. (1989). Scripta Metallurgica, 23(7), 1193-1197.

- Troost, K. Z., Van der Sluis, P., \& Gravesteijn, D. J. (1993). Applied Physics Letters, 62(10), 11101112.

- Wilkinson, A. J. (1996). Ultramicroscopy, 62(4), 237-247.

- Wilkinson, A. J., Meaden, G., \& Dingley, D. J. (2006). Ultramicroscopy, 106(4-5), 307-313.

- Britton, T., \& Wilkinson, A. (2011). Ultramicroscopy, 111(8), 1395-1404.

- Nowakowski, P., Schlenker, J., Ray, M., \& Fischione, P. (2016). Microscopy and Microanalysis, 22(S3), 12-13.

- Nowakowski, P., Bonifacio, C., Ray, M., \& Fischione, P. (2019). Microscopy and Microanalysis, 25(S2), 688-689.

- In ISTFA 2019: Proceedings of the 45th International Symposium for Testing and Failure Analysis. Materials Park, OH: ASM International.

- Nowakowski, P., Wiezorek, J., Bathula, V., Mielo, S., Khanal, S., Bonifacio, C., \& Fischione, P. (2018). Microscopy and Microanalysis, 24(S1), 2182-2183.

- Nowakowski, P., Wiezorek, J., Spinelli, I., Ray, M., \& Fischione, P. (2019). Microscopy and Microanalysis, 25(S2), 534-535. 